\title{
Rotating Balances Used for Fluid Pump Testing
}

\author{
Stephen Skelley ${ }^{1}$ and Andrew Mulder ${ }^{2}$ \\ National Aeronautics and Space Administration, Marshall Space Flight Center, Alabama, 35812
}

\begin{abstract}
Marshall Space Flight Center has developed and demonstrated two direct read force and moment balances for sensing and resolving the hydrodynamic loads on rotating fluid machinery. These rotating balances consist of a series of stainless steel flexures instrumented with semiconductor type, unidirectional strain gauges arranged into six bridges, then sealed and waterproofed, for use fully submerged in degassed water at rotational speeds up to six thousand revolutions per minute. The balances are used to measure the forces and moments due to the onset and presence of cavitation or other hydrodynamic phenomena on subscale replicas of rocket engine turbomachinery, principally axial pumps (inducers) designed specifically to operate in a cavitating environment. The balances are inserted into the drive assembly with power to and signal from the sensors routed through the drive shaft and out through an air-cooled twenty-channel slip ring. High frequency data - balance forces and moments as well as extensive, flush-mounted pressures around the rotating component periphery - are acquired via a high-speed analog to digital data acquisition system while the test rig conditions are varied continuously. The data acquisition and correction process is described, including the in-situ verifications that are performed to quantify and correct for known system effects such as mechanical imbalance, "added mass," buoyancy, mechanical resonance, and electrical bias. Examples of four types of cavitation oscillations for two typical inducers are described in the laboratory (pressure) and rotating (force) frames: 1) attached, symmetric cavitation, 2) rotating cavitation, 3) attached, asymmetric cavitation, and 4) cavitation surge. Rotating and asymmetric cavitation generate a corresponding unbalanced radial force on the rotating assembly while cavitation surge generates an axial force. Attached, symmetric cavitation induces no measurable force. The frequency of the forces can be determined a priori from the pressure environment while the magnitude of the hydrodynamic force is proportional to the pressure unsteadiness.
\end{abstract}

\section{Nomenclature}

$F_{R O T}=$ frequency of oscillation in the rotating frame [Hertz]

$F_{L A B}=$ frequency of oscillation in the laboratory frame [Hertz]

$k$

$=$ circumferential wave number [forward rotation if $\mathrm{k}<0$, backward if $\mathrm{k}>0$ ]

$=$ shaft rotational frequency $[$ Hertz $]$

\section{Introduction}

Marshall Space Flight Center (MSFC) has developed and demonstrated two measurement devices for sensing and resolving the hydrodynamic forces and moments on rotating fluid machinery. Of primary interest are the shaft and/or bearing loads that result from operating in the presence of cavitation and the orientation, amplitude, and frequency content of these hydrodynamic loads. One force sensor and preliminary experimental results were previously described ${ }^{1}$ and the new device and work since then is described here. Emphasis is placed on the data acquisition and processing and the various in-situ verifications that are performed to correct for known system effects are described. These system effects include but may not be limited to mechanical imbalance, the "added mass" of immersion in water, buoyancy, mechanical resonance, and electrical offsets or phase effects.

\footnotetext{
${ }^{1}$ Aerospace Engineer, Fluid Dynamics Branch, Mail Code ER42, non-member.

2 Aerospace Engineer, Fluid Dynamics Branch, Mail Code ER42, non-member.
} 
Table 1. Rotating Balance Full Scale Ranges

\begin{tabular}{|lcc|}
\hline & CDDF \#1 & CDDF \#2 \\
\hline Cal Matrix & $6 \times 12$ & $6 \times 84$ \\
\hline Normal Force & $\pm 50(0.58 \%)$ & $\pm 100(0.17 \%)$ \\
\hline Side Force & $\pm 50(0.61 \%)$ & $\pm 100(0.48 \%)$ \\
\hline Axial Force & $\pm 500(0.48 \%)$ & $\pm 1000(0.15 \%)$ \\
\hline Pitch Moment & $\pm 300(0.46 \%)$ & $\pm 600(0.30 \%)$ \\
\hline Yaw Moment & $\pm 300(0.34 \%)$ & $\pm 600(0.23 \%)$ \\
\hline Roll Moment & $\pm 600(0.26 \%)$ & $\pm 1200(0.14 \%)$ \\
\hline
\end{tabular}

Three of the most common forms of cavitation were observed in water tests of two rocket engine pump inducer designs: 1) attached, symmetric cavitation such as tip vortex and alternate blade cavitation, 2) rotating cavitation, and 3) attached, asymmetric cavitation. By simultaneously acquiring highfrequency pressure data (in the laboratory frame) and force and moment data (in the rotating frame), the tests confirmed that the pressure imbalance caused by rotating cavitation and attached, asymmetric cavitation does generate a unbalanced radial load on the rotating assembly and that the magnitude and orientation of this additional load vector changes with vapor generation.

Finally, based on the available data, a general hydrodynamic force to pressure unsteadiness relationship is proposed.

\section{Rotating Balances}

MSFC has two rotating balances designated CDDF \#1 and CDDF \#2 (Fig. 1) and each consists of a series of instrumented stainless steel flexures arranged around the axis of rotation capped with solid mating rings on the metric and non-metric ends. The force sensors are direct read balances where the bridge outputs are proportional to the applied orthogonal forces (normal, side, and axial) and moments (pitch, yaw, and roll). Semiconductor type, unidirectional strain gauges are arranged into 6 bridges consisting of from 8 to 16 individual gauges. A polyurethane sealant (Micro Measurements M-Coat-A) was applied to the gages as a protective coating followed by several waterproof layers of chloroprene rubber (Tokyo Sokki Kenkyujo N-1). Full-scale ranges of the two balances are summarized in Table 1 along with the percent full-scale errors (2-sigma) from the most recent calibrations. Units are pounds-force or inch-pounds-force. Modern Machine \& Tool of Newport News, Virginia designed, instrumented, and calibrated CDDF \#1. Triumph Aerospace Force Measurement Systems of San Diego, California designed, instrumented, and calibrated CDDF \#2. Micro Craft, Inc. of Tullahoma, Tennessee manufactured both balances.

\section{Experimental Setup and Test Procedure}

The rotating balance is installed between the main test rig drive shaft and test inducer (Fig. 2). The sensor and inducer are joined with a short stub shaft for concentricity control. The entire assembly is submerged in degassed water and the rotating balance hollow core is flooded to purge any trapped air pockets. A small amount of fluid is routed through the device to stabilize the bridge temperatures and minimize thermal drift (less than 1\% full-scale per degree Fahrenheit on all channels). Cabling between the sensor and slip ring is routed through the drive shaft, coupling, and torque meter shaft. All the rotating components are balanced to minimize the radial load associated with any mechanical imbalance.

Power to and signal from the rotating balance is routed through a 20-channel air cooled slip ring (Michigan Scientific Model SR20M/E512/X/ER) attached to the exposed end of the drive assembly (Fig. 3). A pair of amplifiers located on the rotating side of the slip ring (Michigan 


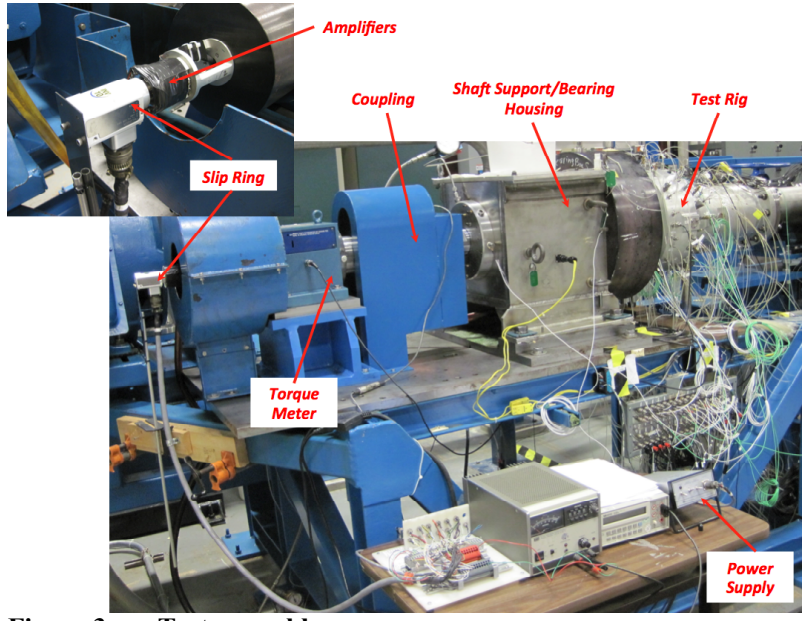

Figure 3. Test assembly.

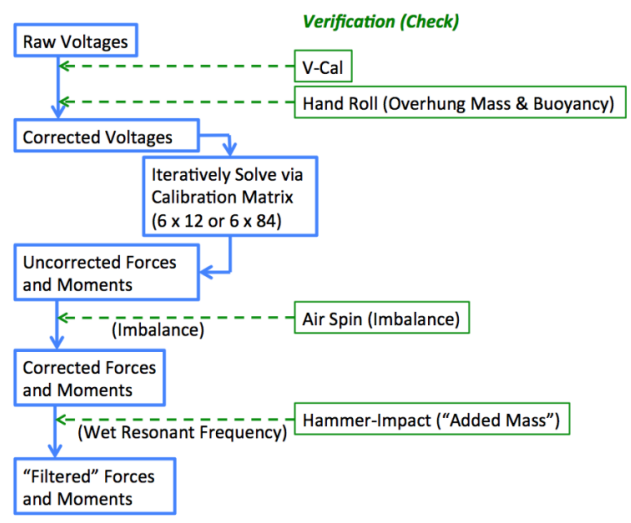

Figure 4. Force and moment data reduction process.

Scientific Model AMP-SG3-U2-5) plus a stationary power supply (Michigan Scientific Model PS-AC) completes the electrical circuit. The amplifiers provide a gain of 100, boosting the signal before it passes through the slip ring.

The data acquisition machine is a 96-channel system withVXI Technology VT1432A digitizer boards. These boards provide 16-bit resolution, approximately $1 \mathrm{MegaOhm}$ input impedance, a common mode rejection ratio greater than 45 decibels, and anti-aliasing filtering. The boards can operate at several different input dynamic range settings, from 0.1 Volts up to 20 Volts. The data recording system is controlled via a computer with a program written in Matlab. The program includes a graphical interface so that the user can easily set sample rate, dynamic range, $\mathrm{AC} / \mathrm{DC}$ coupling, and also observe dynamic data signals in close to real time. High frequency data are acquired at a sample rate of 20480 Hertz per channel (approximately 205 samples per revolution at 6000 revolutions per minute).

Data are recorded continuously while continuously varying either speed or inlet pressure. During a pressure ramp, for example, shaft speed and volumetric flow rate are held constant (for a constant flow coefficient) while inlet pressure is slowly reduced from approximately atmospheric pressure until complete cavitation breakdown is reached (typically around 2-3 pounds per square inch absolute). During a speed ramp the inlet pressure is held constant while the speed is continuously varied across the values of interest. Again, flow coefficient remains approximately constant since the test loop resistance remains constant. Quasi-steady test conditions such as volumetric flow rate, shaft speed, water temperature, etc are recorded by the data acquisition system along with the high frequency pressures and rotating balance forces and moments.

\section{In-Situ Verifications}

The data reduction process by which the sensed voltages are converted to forces and moments is shown schematically in Fig. 4. Four verifications are performed for each test setup, and two of the four (V-CAL and Hand Roll) are performed regularly, as often as several times per day.

\section{A. V-Cal}

The calibration is reported at the end of the cable so the effects of the amplifiers, slip ring, additional cabling (to the data acquisition system), and voltage in must be quantified. First, the voltage at the terminal end of the rotating balance cable is recorded for each channel with the entire electrical system connected and powered. Voltages acquired in test are scaled by the ratio of the measured to calibration reference (5 Volts DC). Next, with the balance cable disconnected a series of known voltages are injected and the values recorded at the data acquisition system. A linear fit of the measured to input is applied to subsequent measurements. This procedure is performed daily and the values recorded and tracked during the test. 


\section{B. Hand Roll}

An in-situ electrical tare is acquired by slowly rotating the entire shaft assembly by hand through 2-3 complete revolutions with the entire system powered (Fig. 5). The mean of the peak-to-peak output on each channel is logged as the channel bias and subtracted from subsequent voltage measurements. The zero-to-peak outputs of the radial components (Normal and Side Force) equal the overhung mass and this value should remain constant throughout the test. The difference in the submerged and dry tare values equals the buoyancy, which is readily compared to the calculated volume of the submerged components (Fig. 6). Hand rolls are typically done multiple times a day as changes in channel bias values are the first indicator of problems with the sensor, slip ring, or other electrical components. Bias also includes any thermal drift effects.

\section{Air Spin}

To quantify the residual mechanical imbalance the test rig is spun in air from idle up to approximately 6000 revolutions per minute with all water purged from the test rig. Only two radial loads should be sensed by the rotating balance (expressed as the resultant of side and normal forces): 1) a load at zero Hertz with a magnitude proportional to the residual mechanical imbalance (Fig. 7) and 2) a load at a frequency equal to the rotational speed with a magnitude proportional to the overhung mass (gravity). A secondary output of the air spin is the resonant frequency or critical speed of the dry rotating assembly. At zero speed where the rotating and laboratory reference frames are coincident these are equal. In the rotating frame, however, the critical speed splits into forward and backward propagating modes separated by two times the operating speed and these two modes are detectable by the rotating balance (Fig. 8). The air spin is usually performed at the start and end of a test series. The imbalance is vectorially subtracted from any measured radial force at zero Hertz.

\section{Hammer-Impact}

The overhung mass reduces the system resonant frequency from the sensor-only value roughly consistent with a single degree of freedom system (Fig. 9). A simple impact hammer test with the test rig dry and filled with degassed water provides an indication of the in-situ resonant frequencies of each component (Fig. 10). The air versus water comparison from the hammer impact test should be consistent with empirical predictions for the "mass-added" effect of submersion. The hammer impact test is typically done at the start of a new test series. Force and moment amplitudes will be conservative at frequencies below and approaching resonance in that the structural amplification will be additive. Measured forces and moments above resonance, however, will be affected by these non-linear system effects, resulting in unreliable amplitudes and phase information. The hammer impact test establishes the per-component functional bandwidth of the rotating balance.

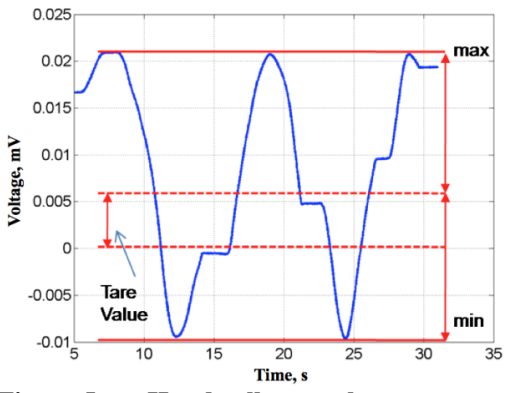

Figure 5. Hand roll example.

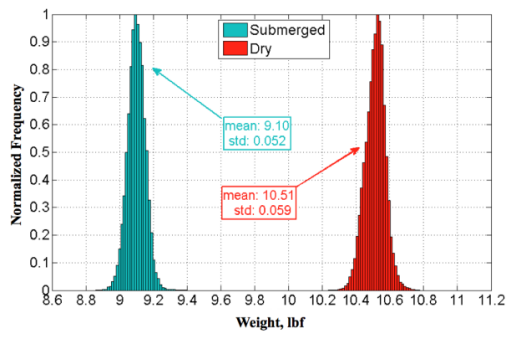

Figure 6. Buoyancy is the dry minus submerged weight.

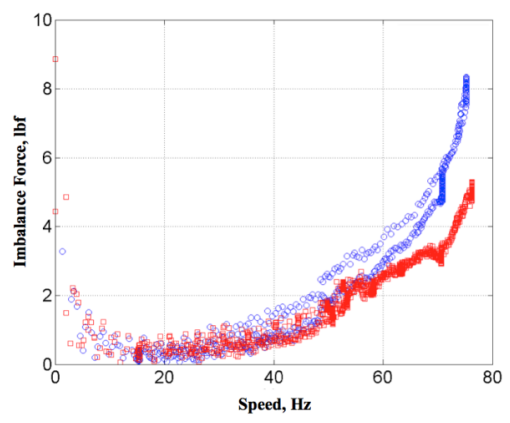

Figure 7. Imbalance versus speed.

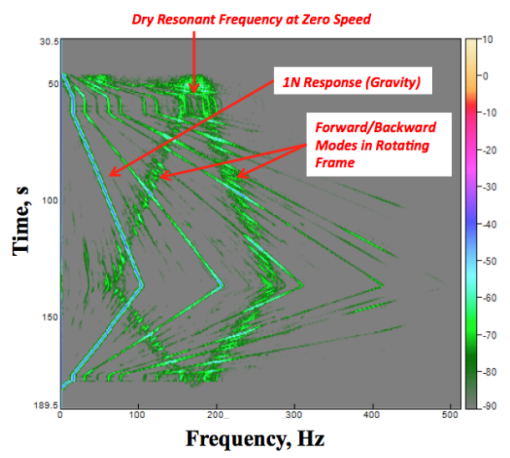

Figure 8. Resonant frequency of side force at speed. 


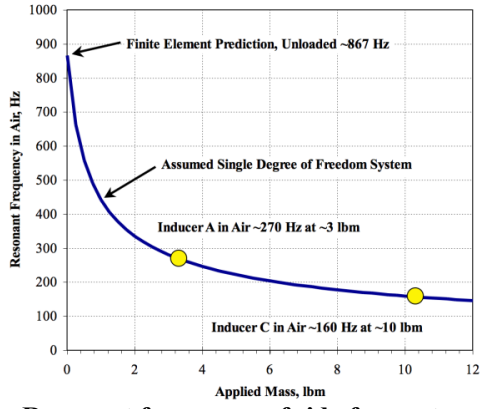

Figure 9. Resonant frequency of side force at zero speed versus overhung mass for CDDF \#2.

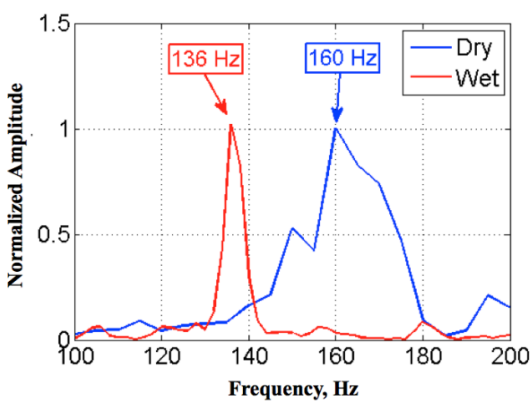

Figure 10. Resonant frequency of side force at zero speed in air and water.

\section{Cavitation-Induced Forces}

The structure of a cavitation-induced oscillation can be extracted from a sufficiently spaced high frequency pressure sensor array. The forces and moments from the rotating balance are spatially integrated values and are essentially point loads on the shaft that can be applied to the structure, seals, and bearings. In combination, these views in the laboratory (pressure) and rotating (forces) frames provide a unique insight into the environment while operating in the presence of cavitation. In particular, rocket engine pump inducers have traditionally exhibited three basic cavitation types: 1) attached, symmetric cavitation, 2) rotating cavitation, and 3) attached, asymmetric cavitation. Some pumps may experience all types, depending on operating conditions and proximity to complete breakdown. Examples of these types as well as auto-oscillation or "cavitation surge" and the hydrodynamic forces associated with each will be described.

The frequency at which an oscillation in the laboratory frame should appear in the rotating frame is described by $F_{R O T}=F_{L A B}+k N$. Higher-order type cavitation with multiple vapor cells and large values of $\mathrm{k}$ will result in force oscillations well above the rotating balance's resonant frequency and they cannot be accurately quantified with the device. For reporting here fluctuating pressure amplitude is normalized by the dynamic pressure based on the inducer leading edge tip. Hydrodynamic forces are normalized by the same dynamic pressure multiplied by the cylindrical area swept between the leading and trailing edge tips. Frequencies are normalized by the shaft speed (N). Spectrogram amplitudes are decibels relative to 1 pound per square inch.

\section{A. Attached, Symmetric Cavitation}

For attached, symmetric modes such tip vortex cavitation, the relationship $F_{R O T}=F_{L A B}+k N$ indicates that any resulting non-rotating, on-rotor radial force will appear as a static load on the balance. No measurable change in the zero-frequency force magnitude or direction for either inducer A (2-blade) or inducer C (4-blade) while operating during attached, symmetric cavitation was detected, indicating insufficient asymmetry to generate a measurable radial load (Fig. 11 and Fig. 12). The $1 \mathrm{~N}$ oscillation in Fig. 12 is the weight of the test components.

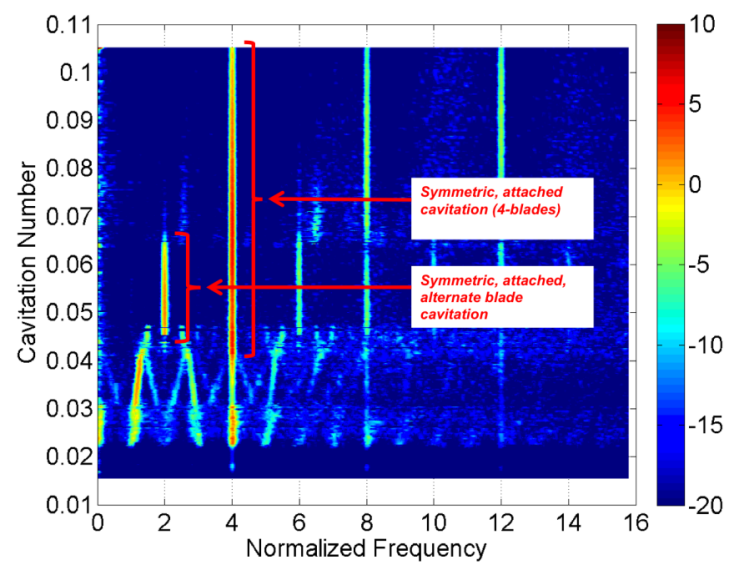

Figure 11. Normalized fluctuating pressure versus normalized frequency and cavitation number, Inducer $\mathbf{C}$.

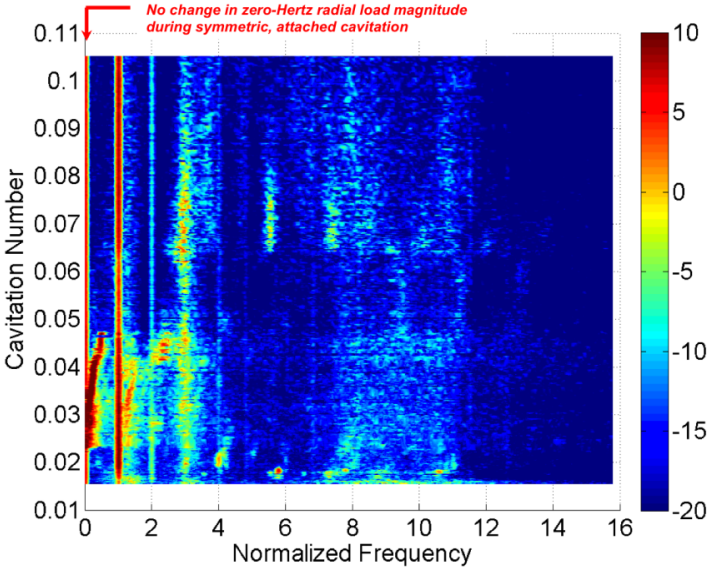

Figure 12. Normalized radial force versus normalized frequency and cavitation number, Inducer $\mathrm{C}$. 


\section{B. Rotating Cavitation}

Rotating cavitation may be described as one or more vapor cells propagating around the periphery of the inducer, usually in the direction of rotation, at frequencies just above the inducer speed. For inducer $\mathrm{C}$ the pressure oscillation appears around a normalized frequency of $1.5 \mathrm{~N}$ and slows as cavitation number is decreased to approximately $1.1 \mathrm{~N}$ with $\mathrm{k}=-1$ (Fig. 13). The force associated with rotating cavitation is just above zero Hertz in the rotating frame (Fig. 14). Although the fluctuating pressure amplitude during rotating cavitation was less than that associated with blade-passing, the asymmetric pressure distribution induces a significant radial load which must be ultimately reacted by the bearing and or shaft support structure. Tracking the oscillation in the laboratory and rotating frames during a pressure ramp at constant flow coefficient and speed (Fig. 15) illustrates the typically abrupt onset of rotating cavitation and the sharp increase in radial load resulting from the sudden pressure rebalancing.

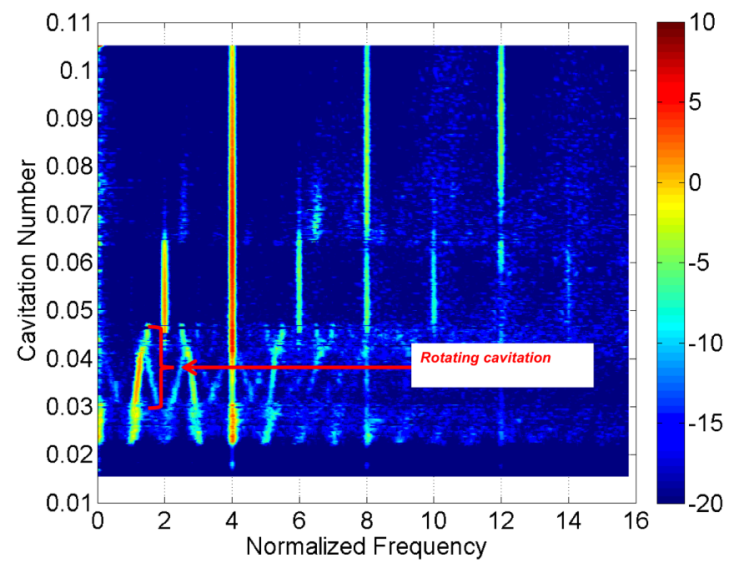

Figure 13. Normalized fluctuating pressure versus normalized frequency and cavitation number, Inducer $\mathrm{C}$.

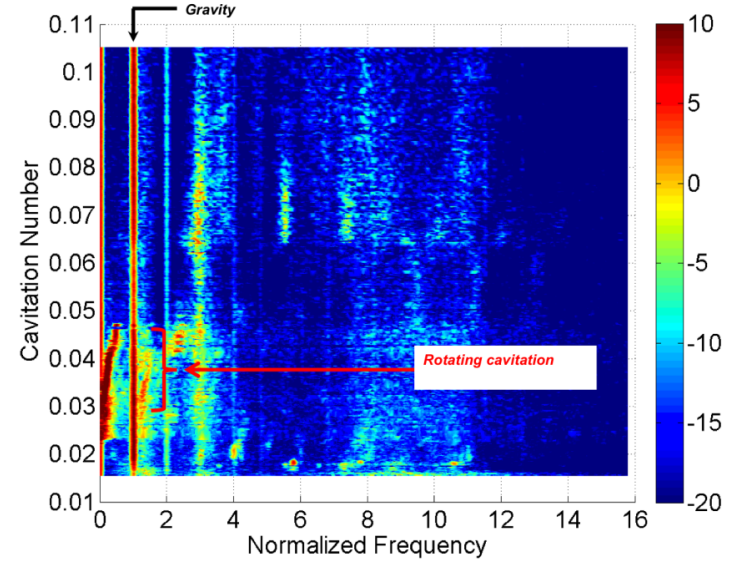

Figure 14. Normalized radial force versus normalized frequency and cavitation number, Inducer $\mathrm{C}$.

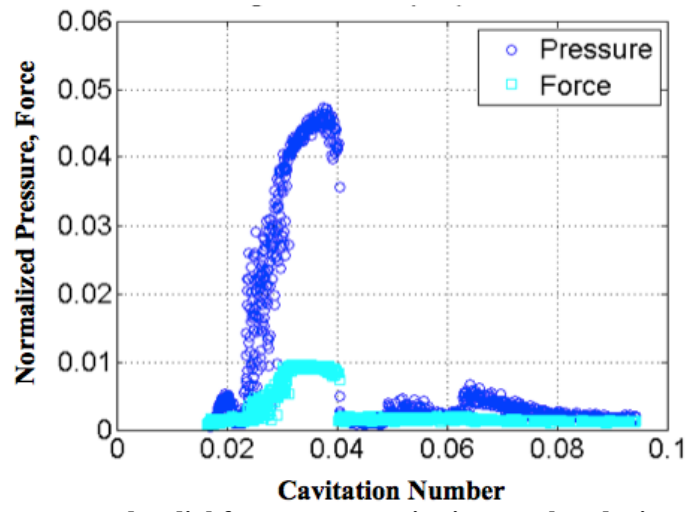

Figure 15. Normalized fluctuating pressure and radial force versus cavitation number during rotating cavitation, Inducer C.

\section{Attached, Asymmetric Cavitation}

Attached, asymmetric cavitation also collapses to $F_{R O T}=0$ and again, the radial force magnitude must be extracted from the zero Hertz or DC data. Unlike attached, symmetric cavitation, the non-rotating pressure distribution during attached, asymmetric cavitation generates a significant hydrodynamic load resembling a mechanical imbalance that may exacerbate the inherent imbalance or increase the rotating assembly radial deflection. For inducer A with two blades and $\mathrm{k}=-1$, the fluctuating pressure at $1 \mathrm{~N}$ (Fig. 16) corresponds to a normalized radial force (Fig. 17) comparable to that measured with inducer $\mathrm{C}$ during rotating cavitation. Moreover, the balance data shows a slow rotation of the center of pressure with decreasing cavitation number, indicating the center of pressure moves downstream with increasing vapor generation near the inducer leading edge. 


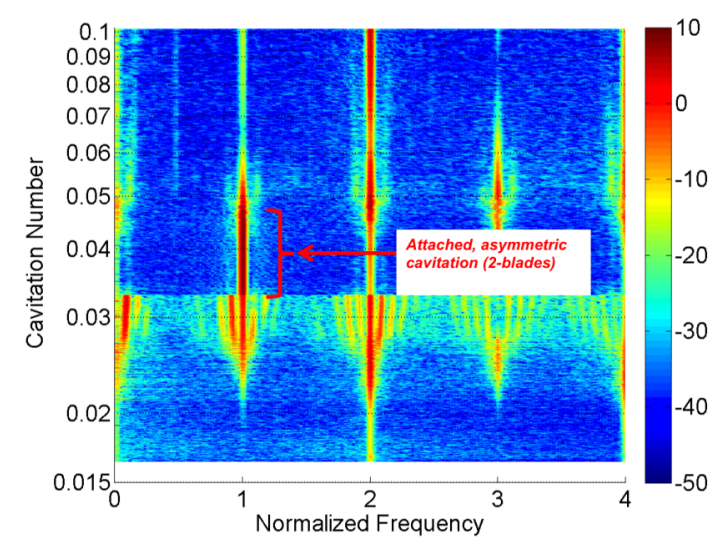

Figure 16. Normalized fluctuating pressure versus normalized frequency and cavitation number, Inducer $A$.

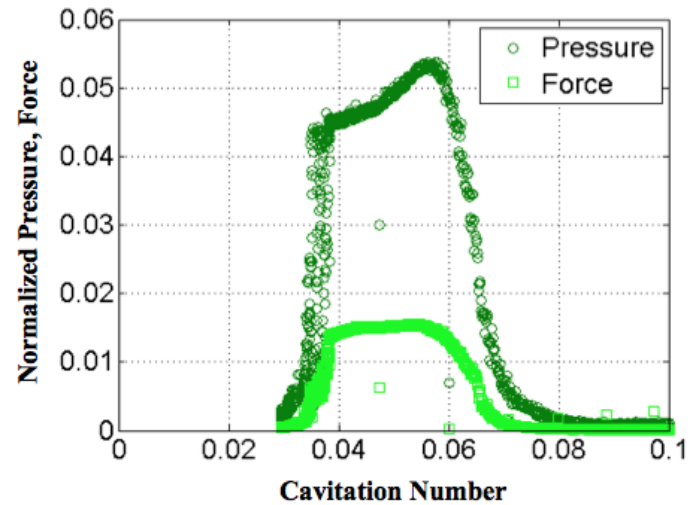

Figure 17. Normalized fluctuating pressure and radial force versus cavitation number during attached, asymmetric cavitation, Inducer A.

\section{Cavitation Surge}

Cavitation surge or auto-oscillation is a planar oscillation typically caused by cavitation-induced changes to the system's one-dimensional, low-frequency stability. As a planar oscillation with $\mathrm{k}=0$, the pressure oscillation and corresponding force(s) should appear at the same frequency in both reference frames. Surge was observed with inducer $\mathrm{C}$ resulting in a hydrodynamic load in the axial direction (Fig. 18).

\section{E. Hydrodynamic Force to Pressure Unsteadiness Correlation}

High frequency response pressure sensors are the simplest tool for identifying and characterizing cavitation phenomena and, as such, a gross correlation between the pressure unsteadiness and potential hydrodynamic force would be useful in assessing a new design without actually measuring the induced forces. Water testing at MSFC indicates that such a relationship may exist with one significant caveat: pressure unsteadiness is highly sensitive to axial location so analogous sensor locations are critical if hydrodynamic load is to be inferred. The previouslydescribed results are combined in Fig. 19, suggesting that the resultant force is less sensitive to the type of cavitation oscillation than the magnitude of the oscillation. Any significance of the slope of force to pressure unsteadiness is unknown and the correlation is presented here merely for guidance. Results from additional pumps will be added as they become available.

\section{References}

${ }^{1}$ Skelley, S., "Inducer Hydrodynamic Forces in a Cavitating Environment," Proceedings of the Heat Transfer/Fluids Engineering Summer Conference, Vol 3, ASME, New York, 2004, pp. 37-45.

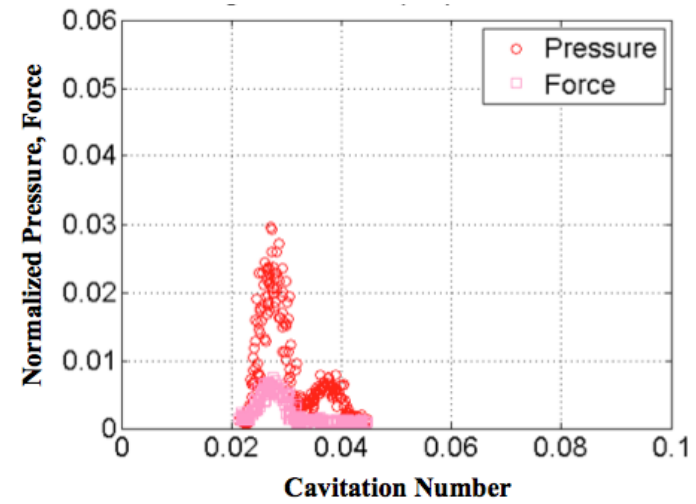

Figure 18. Normalized fluctuating pressure and axial force versus cavitation number during cavitation surge, Inducer $C$.

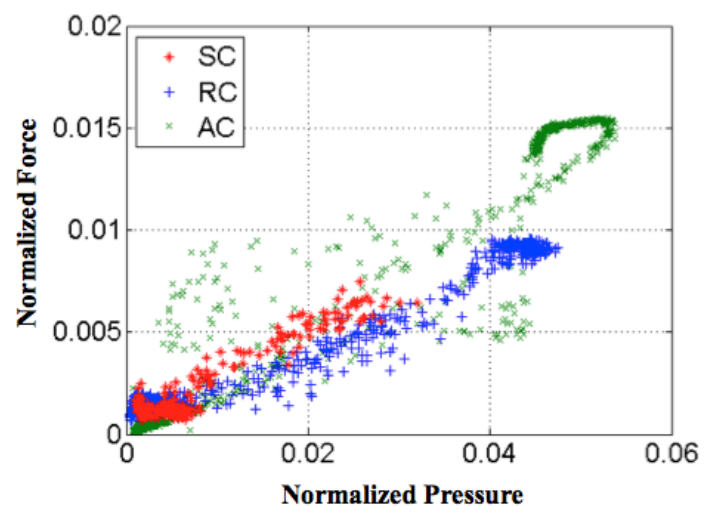

Figure 19. Normalized force versus normalized fluctuating pressure. Inducer $\mathrm{C}$ cavitation surge (SC) and rotating cavitation (RC). Inducer $A$ attached, asymmetric cavitation (AC). 